\title{
A meta-analysis and systematic review of intracranial pressure monitoring on severe craniocerebral injury
}

\author{
Huijun Wang, Yi He, Ruofei Liang, Xuan Wu, Long Zhao, Juan Yang, Xiaoping Tang \\ Neurosurgery Department, the Affiliated Hospital of North Sichuan Medical College, Nanchong, China \\ Contributions: (I) Conception and design: H Wang, X Tang; (II) Administrative support: Y He; (III) Provision of study materials or patients: H Wang, \\ Y He, R Liang, X Wu, L Zhao; (IV) Collection and assembly of data: All authors; (V) Data analysis and interpretation: R Liang, X Wu, L Zhao, J \\ Yang, X Tang; (VI) Manuscript writing: All authors; (VII) Final approval of manuscript: All authors. \\ Correspondence to: Xiaoping Tang. 36 Yutan Street, Shunqing District, Nanchong, China. Email: $915185701 @ q q . c o m$.
}

\begin{abstract}
Backgrounda A meta-analysis was carried out to study the effect of intracranial pressure monitoring on the prognosis of severe craniocerebral injury and to provide reference for treating craniocerebral diseases.

Methods: A Boolean logic search method was adopted, and "intracranial pressure monitoring", "craniocerebral injury", "prognosis", and "brain injury" were set as search terms. The literature searched included PubMed, Medline, and the China National Knowledge Internet (CNKI), and literature that set non-intracranial pressure monitoring as a control for comparative research was screened. RevMan was then employed to perform the meta-analysis.

Results: 13 studies were included, most of which were of medium and high quality (low-risk bias). The results showed that no heterogeneous in-hospital mortality was found between groups $\left(\chi^{2}=0.76, \mathrm{I}^{2}=0 \%\right.$, $\mathrm{P}=0.98$ ) and the hospital mortality of experimental group was dramatically inferior to control (ctrl) group $(\mathrm{Z}=3.69, \mathrm{P}=0.0002)$. Heterogeneity was found in the probability of favorable functional prognosis between groups $\left(\chi^{2}=8.01, \mathrm{I}^{2}=50 \%, \mathrm{P}=0.09\right)$ and the probability of favorable functional prognosis in experimental group was remarkably superior to that in ctrl group $(\mathrm{Z}=2.48, \mathrm{P}=0.01)$. Incidence of renal failure was not heterogeneous between groups $\left(\chi^{2}=3.17, \mathrm{I}^{2}=0 \%, \mathrm{P}=0.53\right)$ and the incidence of renal failure in experimental group was notably inferior to ctrl group $(\mathrm{Z}=2.71, \mathrm{P}=0.007)$. Finally, the incidence of lung infection in the two groups was heterogeneous $\left(\chi^{2}=6.35, \mathrm{I}^{2}=37 \%, \mathrm{P}=0.17\right)$ and incidence of lung infection in experimental group was dramatically lower versus ctrl group $(\mathrm{Z}=2.13, \mathrm{P}=0.03)$.
\end{abstract}

Discussion: Our results showed Intracranial pressure monitoring can effectively reduce in-hospital mortality and the incidence of infection in patients with severe craniocerebral damage and enhance the functional prognosis of patients. Due to the inclusion criteria imposed in this study, the number of included articles was limited, and in future analyses the sample size should be increased to prevent bias.

Keywords: Intracranial pressure; craniocerebral injury; functional prognosis; infection

Submitted Mar 04, 2021. Accepted for publication May 20, 2021.

doi: 10.21037/apm-21-897

View this article at: http://dx.doi.org/10.21037/apm-21-897

\section{Introduction}

Severe craniocerebral injury is a type of disorder of the central nervous system that regulates the hypothalamuspituitary-adrenal cortex axis and leads to increased secretion of catechol, glucocorticoid and glucagon, decreased insulin secretion, enhanced body catabolism, and increased energy consumption. Such high metabolic reactions consume energy stored in the body, damage body tissue and organs, and complicate infections $(1,2)$. Craniocerebral injury is the second most common form of systemic injury (second only to limb injuries) yet has the highest mortality rate $(3,4)$. Around 500,000 craniocerebral injuries occur in China each year, and the death toll is about 100,000 , 
making it the fourth leading cause of death after heart disease, malignant tumors, and cerebrovascular accidents $(5,6)$. Based on its site and resulting pathological changes, craniocerebral injury is classified into an open and closed type. A diagnosis of open craniocerebral injury is mainly based on rupture of the dura mater, cerebrospinal fluid outflow, cranial cavity, and external communication $(7,8)$. Severe headache, frequent vomiting, and a sharp increase in intracranial pressure may be a sign of brain herniation. In patients with impaired consciousness, from being able to change the lying position on their own or being able to change their head position by themselves when vomiting, they are regarded as aggravated. Continuous intracranial pressure monitoring with an intracranial pressure monitor can detect intracranial pressure as soon as possible, and timely and effective treatment can prevent the formation of brain herniation and avoid deterioration of the condition $(9,10)$. In addition, patients with craniocerebral injury will have different degrees of cerebral edema. The increase in intracranial pressure caused by severe cerebral edema is often a fatal factor, and attention should be paid to prevent any increase (11).

Increased intracranial pressure can occur with many conditions and diseases affecting the brain and is present when pressure continues to be above $2.0 \mathrm{kPa}\left(200 \mathrm{mmH}_{2} \mathrm{O}\right)$, resulting in corresponding symptoms and signs $(12,13)$. Intracranial pressure monitoring assesses the pressure of the contents of the cranial cavity on the wall of the cranial cavity through the placement of a detection probe within the skull and the corresponding waveform of intracranial pressure is transmitted to a workstation (14). Analyzing changes in intracranial pressure helps determine the status of brain edema, which in turn helps determine treatment measures and estimate prognosis. This meta-analysis investigates the effect of intracranial pressure monitoring on the prognosis of patients with severe craniocerebral damage. We present the following article in accordance with the PRISMA reporting checklist (available at http://dx.doi.org/10.21037/ apm-21-897).

\section{Methods}

\section{Literature screening}

Boolean logic retrieval was utilized to evaluate the selected related literature sources, and terms including "intracranial pressure monitoring", "craniocerebral injury", "prognosis", "brain injury" were searched. The PubMed, Medline,
EMbase, China Biomedical Literature, CNKI, Wanfang, Weipu, and Google Scholar databases were then searched. All reference lists included in any literature or published reviews were also traced to screen literature not indexed by the database. The search time was from the establishment to October 30, 2020. Literature quality was evaluated according to RevMan 5.2 provided by the Cochrane system and various search terms were combined freely. Moreover, the results were supplemented by contacting related experts and researchers to obtain the most contemporary reports.

\section{Literature inclusion and exclusion criteria}

The included literature met the following criteria: (I) the research objects were patients diagnosed with severe craniocerebral injury; (II) experimental patients were monitored for intracranial pressure; (III) patients in ctrl group did not undergo intracranial pressure monitoring (imaging or clinical symptoms); (IV) the types of studies were randomized clinical trials, prospective cohort or casecontrol studies; (V) Glasgow coma scale (GCS) score of patients was less than 9 points.

Articles characterized by any of the following were excluded: (I) the included subjects had primary acute and chronic cardiopulmonary dysfunction, diabetes, or mental illnesses; (II) literature without randomized controlled experiments; (III) valid data was not provided or literature data was missing; (IV) research objects or data overlapped each other; (V) literature that were repeatedly published or involved insufficient experimental samples.

Two senior experts screened the title, abstract, and full text independently, and three preliminary experiments were performed. Any inconsistent opinions among experts were addressed by consensus following discussion, or through arbitration by a third expert.

\section{Quality evaluation}

Newcastle-Ottawa Scale (NOS) of the Cochrane Collaboration was adopted to evaluate the pathological control studies included. A star system (highest score nine stars) was set to assess the results of study object, case comparison, and comparison between groups. Literature with seven stars and above was deemed as high quality and having low risk bias, and that with one star or no star was deemed as low quality and having high risk bias. Literature with two to six stars was considered medium quality, that is, medium risk bias. 


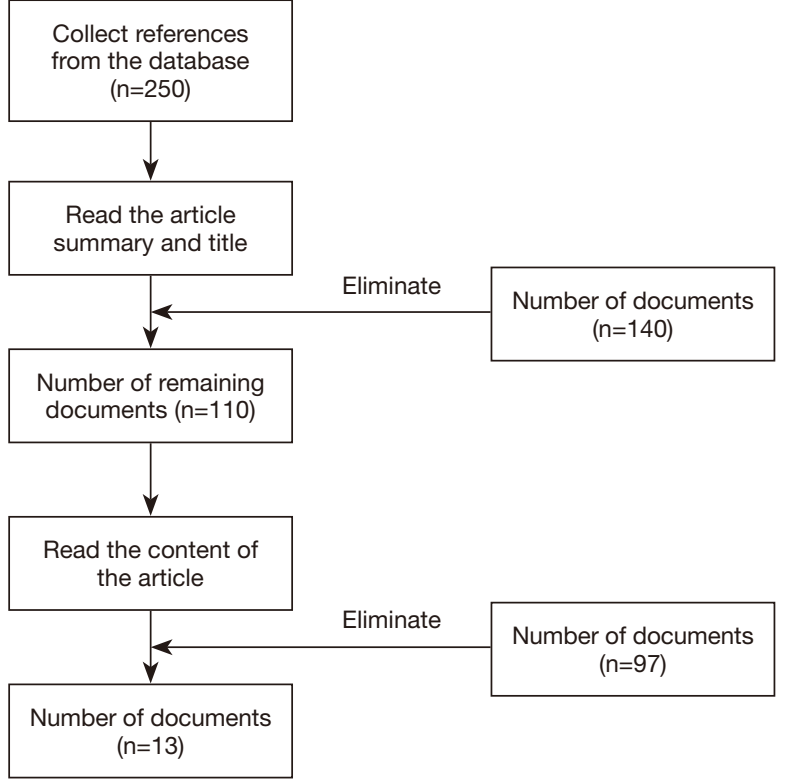

Figure 1 Literature screening process.

\section{Data extraction}

The two experts utilized a unified Excel table to extract data independently. The data extracted included: (I) the first author and publication year; (II) the number of subjects of two groups; (III) the grouping methods and the interventions used in ctrl group and experimental group; (IV) indicators of a prognostic effect of craniocerebral injury, such as hospital mortality, better functional prognosis rate, and lung infection rate.

\section{Statistical analysis}

RevMan 5.3 was employed for meta-analysis and mean difference (MD) or standardized MD (SMD) and a 95\% confidence interval (CI) were adopted to analyze the efficacy of the continuous variables of the research data. The included literatures were first tested for heterogeneity using Q test. The risk of bias assessment chart of RevMan was then utilized to assess the risk bias. Each effect was expressed by a $95 \% \mathrm{CI}$ and when $\mathrm{P}>0.1$ and $\mathrm{I}^{2}<50 \%$, the fixed effects model (FEM) was utilized. Finally, when $\mathrm{P}<0.1$ and $\mathrm{I}^{2}>50 \%$, the random effects model (REM) was utilized.

\section{Results}

\section{Literature screening and NOS scale rating results}

A total of 250 literatures were obtained and 140 were eliminated after the abstracts and titles were read (Figure 1). After the full text of the article was read, a further 97 ones were eliminated, resulting in 13 ones finally selected for meta-analysis. Among the excluded articles, the focus of 46 involved primary acute and chronic cardiopulmonary dysfunction, diabetes, and psychiatric diseases, 21 involved animal experiments, 35 involved repeated research subjects, 66 contained research related information that could not be extracted, 48 involved research indicators of noncraniocerebral injury prognostic indicators, and 21 lacked original data in the research results. Table 1 shows the basic information in the included literature which covered 20002013. Figure 2 depicts the results of the NOS scale rating which shows there were four articles with 7 stars and above, nine with 2-6 stars, and none receiving less than 2 stars or less, indicating all were of medium and high-quality.

\section{Literature risk bias}

Figures 3 and 4 show the literature multiple risk bias evaluation results drawn by the RevMan. Each methodological feature of an article is included, and the evaluation results were input into the software to generate a plot. Random sequence generation (which refers to selection bias), allocation hiding (which refers to selection bias), blinding of result evaluation (which refers to measurement bias), incomplete result data (which refers to follow-up bias), selective reporting (which refers to reporting bias) show obviously low risk bias. Blinding of subjects and researchers (which refers to implementation bias) and other low-risk bias evaluations are also around 50\%. Except for the literature of Griesdale [2010] and Huang [2013], the risk bias of the other articles is obviously low.

\section{In-bospital mortality}

Figure 5 shows the in-hospital mortality between groups in a randomized controlled study. The results of Randall [2012] had the highest proportion of the final combined 
Table 1 Basic information of literature included

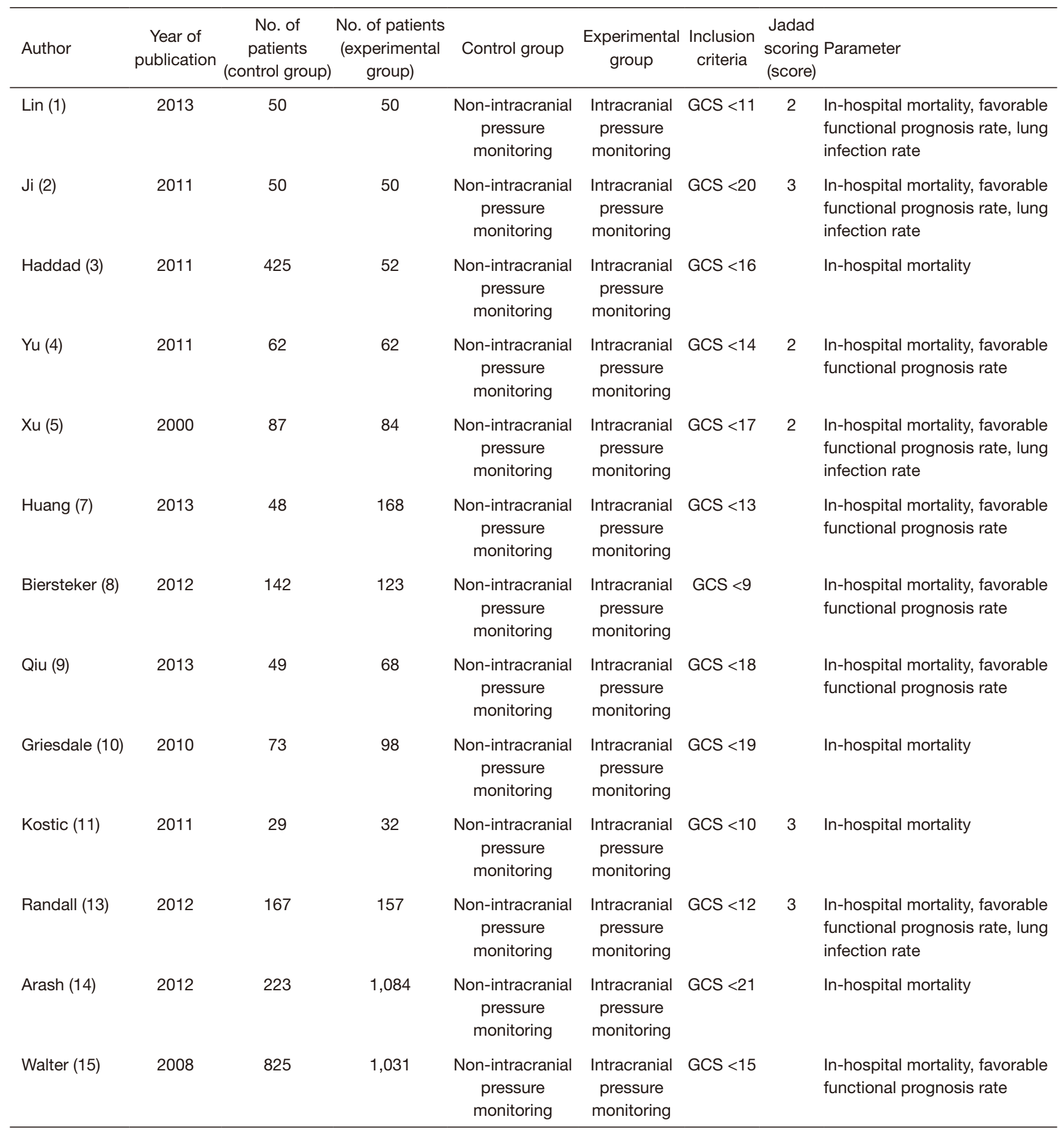


results $(37.3 \%)$, followed by $\mathrm{Xu}[2000](17.0 \%)$, and Lin [2013] (14.0\%). Horizontal line (HL) of 95\% CI of most articles falls to the left of invalid vertical line (IVL). HL of articles crosses IVL, and HL of $95 \%$ CI of a few articles falls to the right of IVL. In the 13 included studies, 445 cases were in ctrl group, and 435 cases were in experimental group. No heterogeneous was indicated in in-hospital mortality between groups $\left(\chi^{2}=0.76, \mathrm{I}^{2}=0 \%, \mathrm{P}=0.98\right)$. The diamond block, which indicates combined effect size, is on the left side of IVL, OR is 0.55 (95\% CI: $0.40-0.75$ ). Therefore, the FEM analysis was implemented, and the inhospital mortality rate of experimental group was shown to be substantially lower relative to that of ctrl group $(\mathrm{Z}=3.69$, $\mathrm{P}=0.0002)$.

Figure 6 presents the funnel plot illustrating inhospital mortality between groups. Circles of literature are concentrated in the top, and the research accuracy is

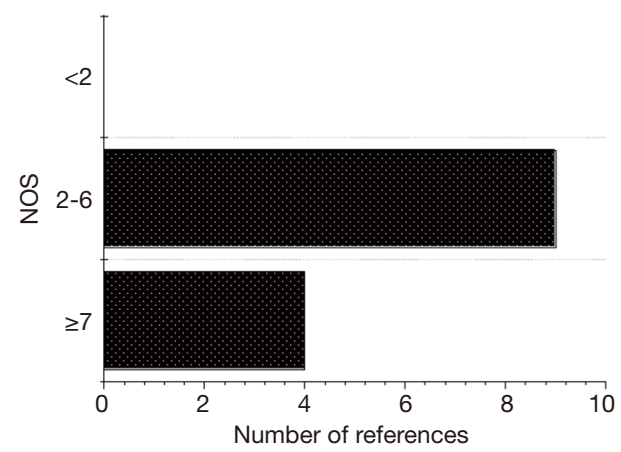

Figure 2 Quality rating results of NOS.

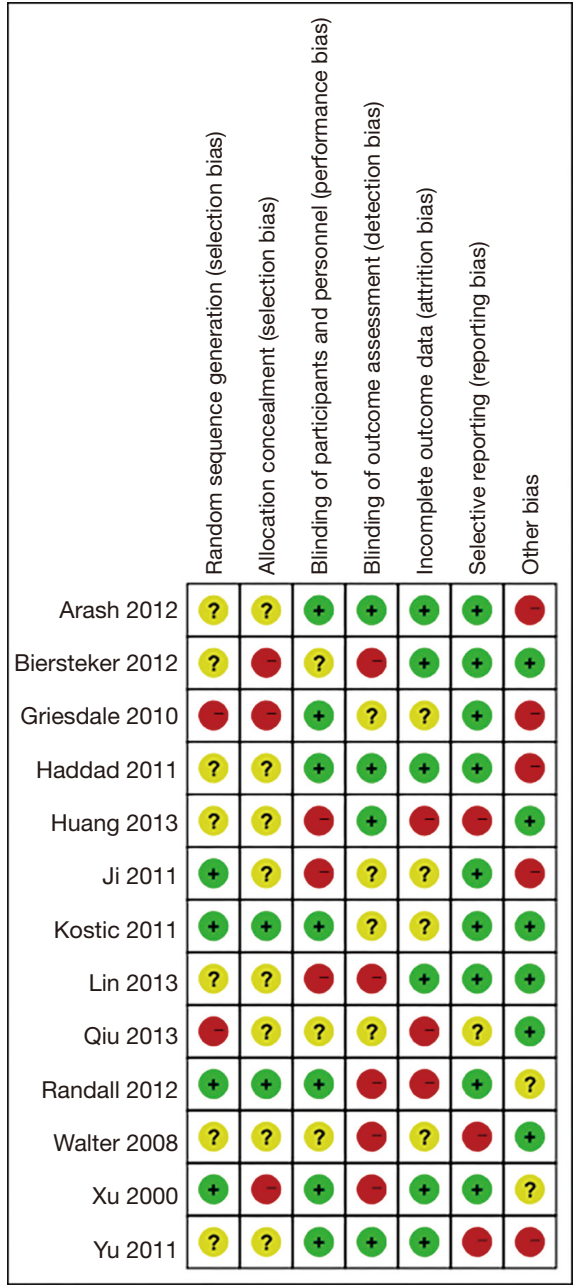

Figure 4 Literature risk bias evaluation results. Red means high risk of bias; yellow means unclear risk of bias; green means low risk of bias.

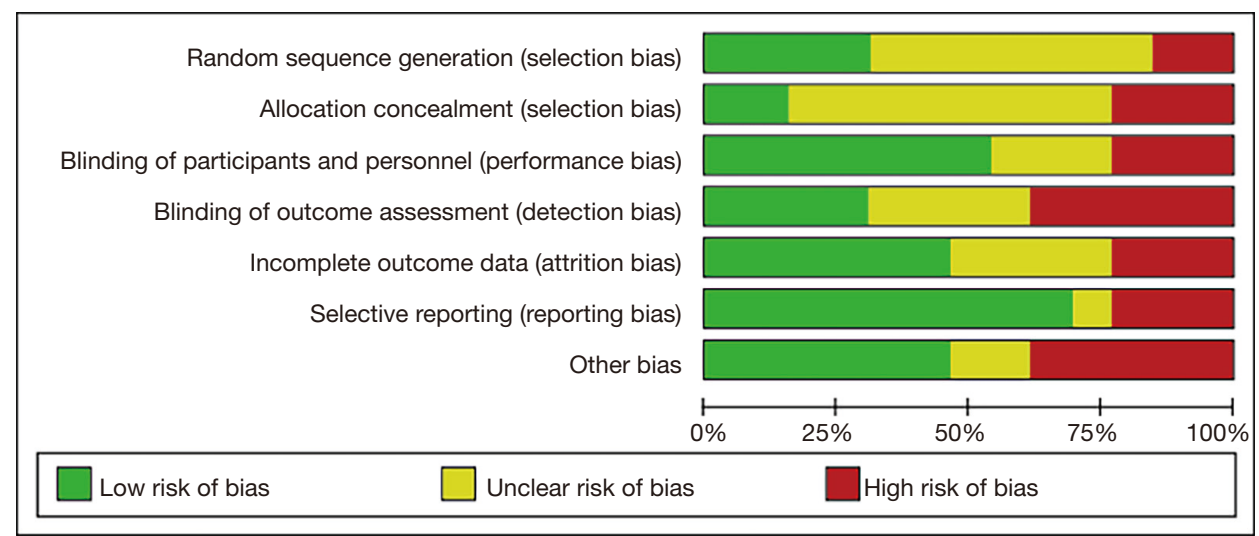

Figure 3 Literature risk bias evaluation results. 


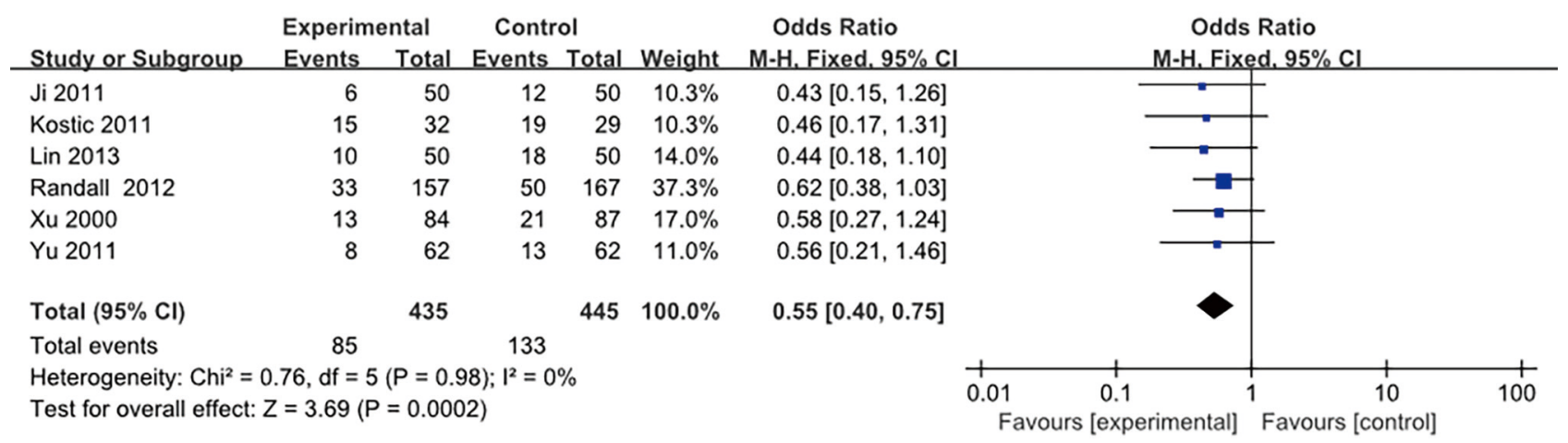

Figure 5 Comparison of in-hospital mortality of patients.

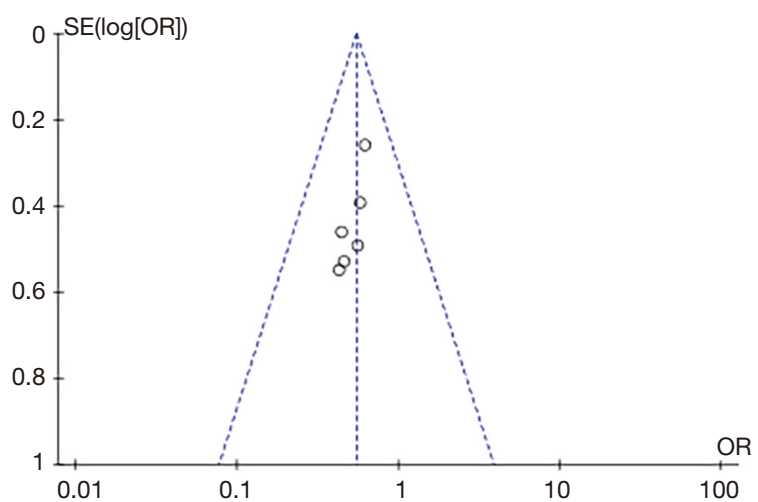

Figure 6 Funnel chart of comparison results of in-hospital mortality. SE (log (OR)): standard error; OR: effect size; they had the same meanings for all the figure below. high. Although circles of literature are distributed on both sides of midline, they are not symmetrical. Therefore, the included literature has publication bias.

\section{Comprebensive incidence}

Figure 7 shows comprehensive incidence of patients in the cohort study. The research of Walter [2008] had the highest proportion of the final combined results (19.7\%), followed by Arash [2012] (18.5\%), and Biersteker [2012] (16.3\%). HL of $95 \%$ CI of most literature falls to the left of IVL. HL of literature crosses IVL, and HL of $95 \%$ CI of a few articles falls to the right of IVL. Among the 13 articles included, 1,785 cases were in ctrl group and 2,624

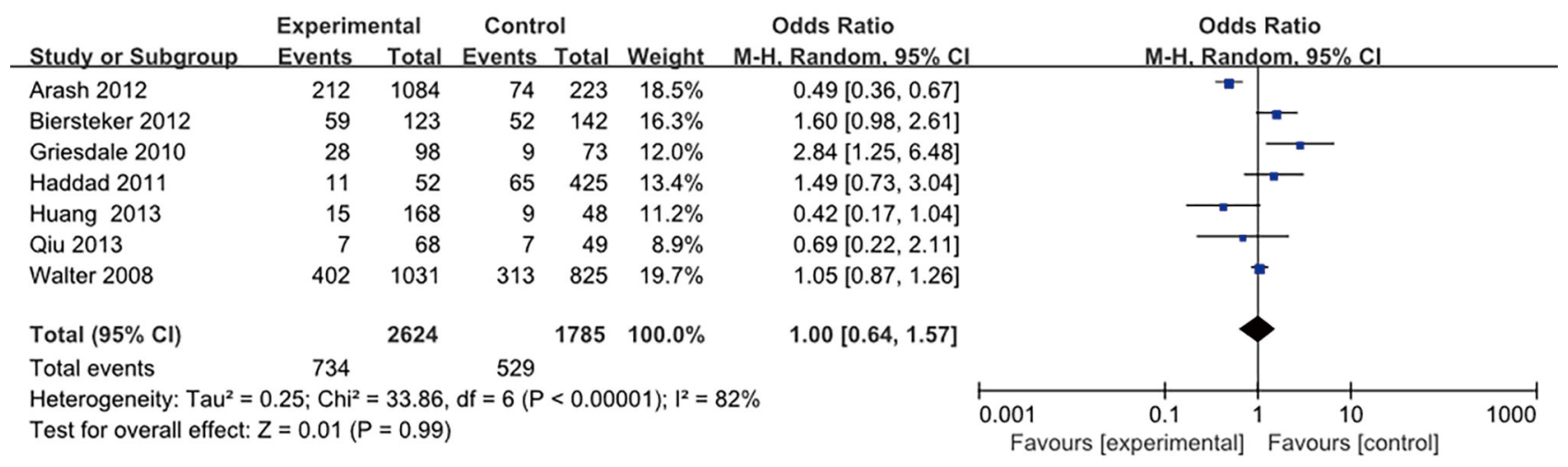

Figure 7 Comprehensive mortality of two groups of patients. 


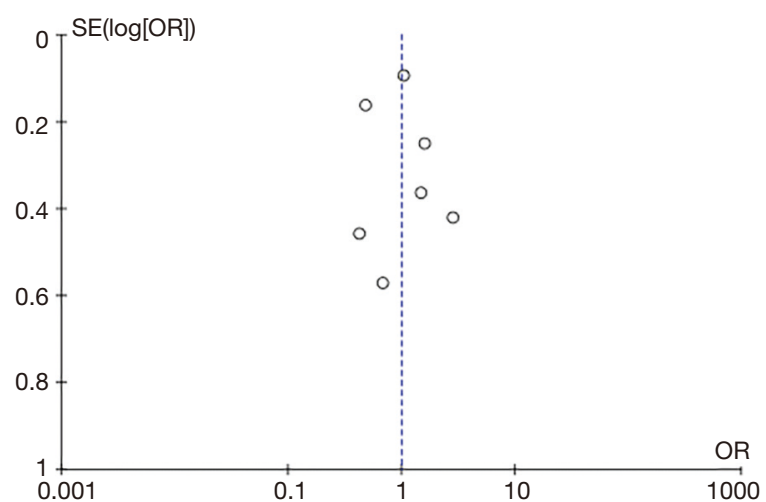

Figure 8 Funnel chart of the comparison result of the comprehensive case and death situation.

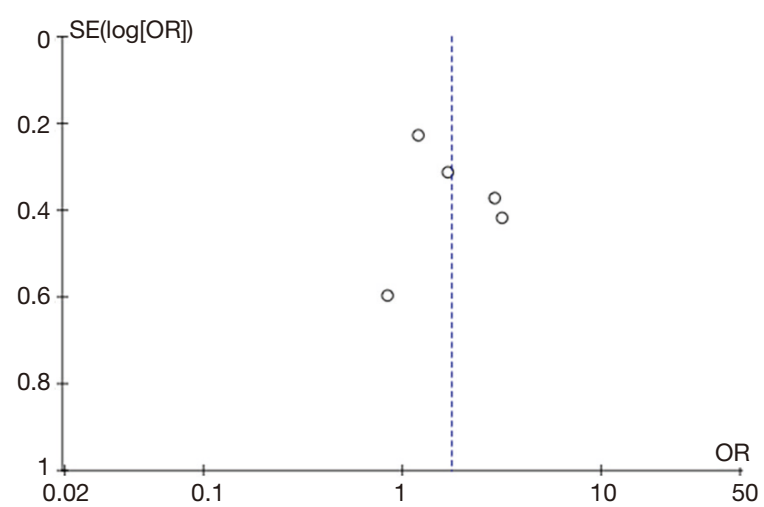

Figure 10 Funnel plot for favorable functional prognosis comparison.

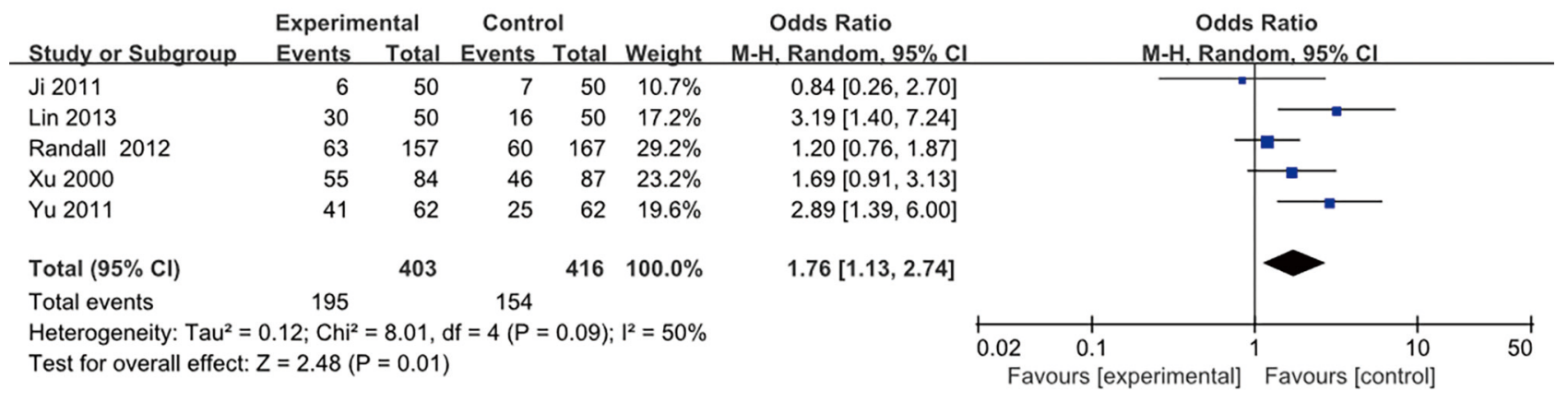

Figure 9 Comparison of probability of favorable functional prognosis.

were in experimental group. There was heterogeneity in the comprehensive incidence of patients between two groups $\left(\chi^{2}=33.86, \mathrm{I}^{2}=82 \%, \mathrm{P}<0.00001\right)$. The diamond block crosses IVL, OR is 1.00 (95\% CI: 0.64-1.57). Therefore, random model analysis was performed, and the results show that no marked difference was revealed in the overall incidence of patients between groups $(\mathrm{Z}=0.01, \mathrm{P}=0.99)$.

Figure 8 presents funnel plot illustrating comprehensive incidence of patients in two groups in the cohort study. Circles of literature are distributed on both sides of midline, and are nearly symmetrical, indicating no publication bias in the included literature.

\section{Probability of favorable functional prognosis}

Figure 9 shows the comparison of the probability of favorable functional prognosis between groups. The research results of Randall [2012] had the highest proportion of the final combined results (29.2\%), followed by $\mathrm{Xu}$ [2000] (23.2\%), and Yu [2011] (19.6\%). HL of $95 \%$ CI of most literature falls to the right of IVL. HL of literature crosses IVL, and HL of $95 \%$ CI of a few articles falls to the left of IVL. In the included 13 articles, 416 cases were in ctrl group and 403 were in experimental group. The probability of favorable functional prognosis between groups was heterogeneous $\left(\chi^{2}=8.01, \mathrm{I}^{2}=50 \%, \mathrm{P}=0.09\right)$. The diamond block is on the right side of IVL, OR is 1.76 (95\% CI: 1.13-2.74). Random model analysis showed that the probability of better functional prognosis in experimental group was remarkably superior to $\mathrm{ctrl}$ group $(Z=2.48$, $\mathrm{P}=0.01)$.

Figure 10 shows funnel plot illustrating probability of favorable prognostic function between groups. Although circles of literature are distributed on both sides of midline, they are not symmetrical. Therefore, the included literature has publication bias. 


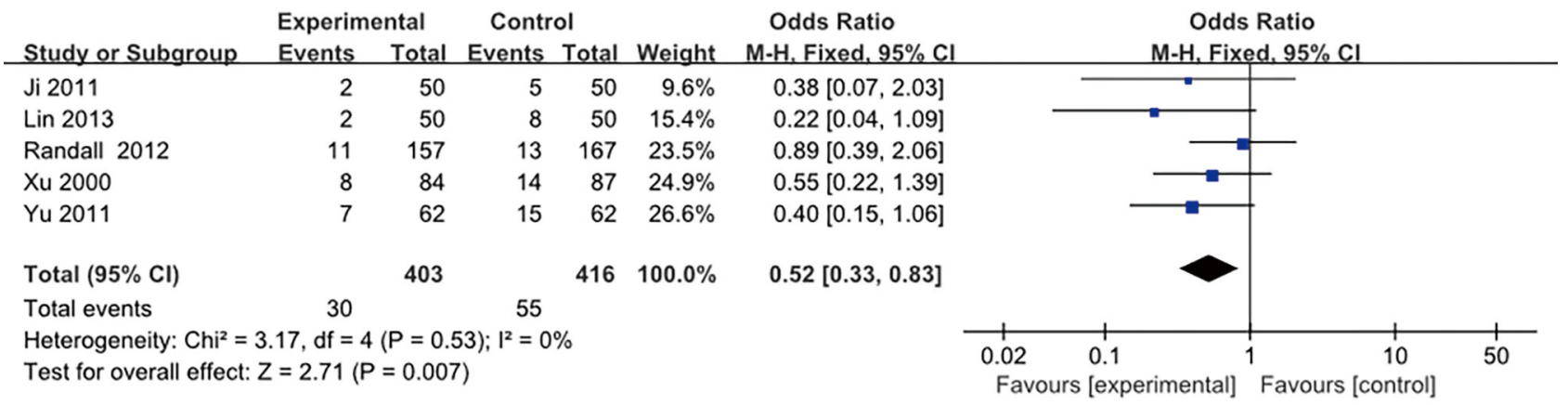

Figure 11 Incidence of renal failure between groups.

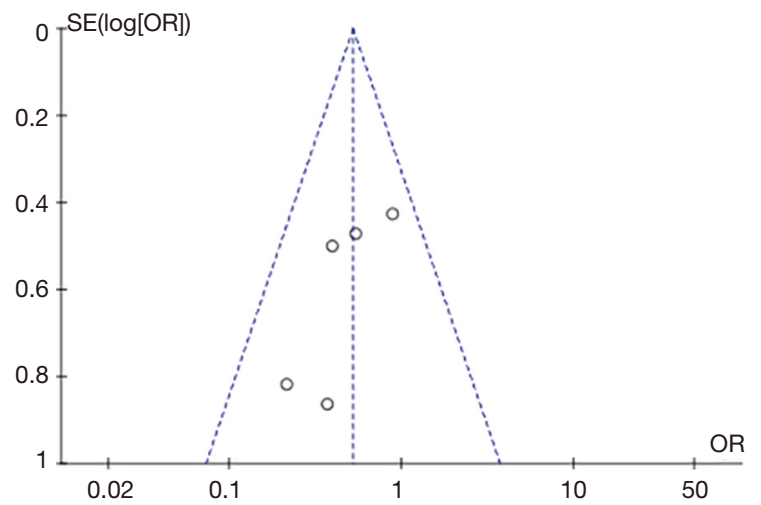

Figure 12 Funnel plot of comparison results of renal failure rate

\section{Incidence of renal failure}

Figure 11 illustrates incidence of renal failure between groups. The research of Yu's [2011] had the highest proportion of the final combined results $(26.6 \%)$, followed by the results of $\mathrm{Xu}$ [2000] (24.9\%), and Randall [2012] (23.5\%). HL of $95 \%$ CI of most literature falls to the left of IVL. HL of literature crosses IVL, and HL of $95 \%$ CI of a few articles falls to the right of IVL. In the included 13 articles, 416 cases were in ctrl group while 403 were in experimental group. The incidence of renal failure between groups was not heterogeneous $\left(\chi^{2}=3.17, \mathrm{I}^{2}=0 \%, \mathrm{P}=0.53\right)$. The diamond block is on the left side of IVL, OR is 0.52 (95\% CI: 0.33-0.83). Therefore, a FEM was adopted for analysis, and the incidence of renal failure in experimental group was notably inferior to that of $\operatorname{ctrl}$ group ( $\mathrm{Z}=2.71$, $\mathrm{P}=0.007)$.

Figure 12 shows funnel plot illustrating incidence of renal failure between groups. Although the circles of literature are found on both sides of midline, they are not symmetrical. Therefore, the included literature has publication bias.

\section{Incidence of lung infection}

Figure 13 presents incidence of lung infection between groups. The research results of Randall [2012] had the highest proportion $(50.4 \%)$ of the final combined results, followed by those of Biersteker [2012] (21.9\%). HL of $95 \%$ CI of most literature falls to the left of IVL. HL of literature crosses IVL, and HL of $95 \%$ CI of a few articles falls to the right of IVL. In the 13 articles included, 496 cases were in ctrl group while 464 were in experimental group. The incidence of lung infection between groups was heterogeneous $\left(\chi^{2}=6.35, \mathrm{I}^{2}=37 \%, \mathrm{P}=0.17\right)$. The diamond block is on the left side of IVL, OR is 0.70 (95\% CI: 0.50 0.97). The FEM was adopted for analysis, and the incidence of lung infection in experimental group was notably inferior to that of ctrl group $(\mathrm{Z}=2.13, \mathrm{P}=0.03)$.

Figure 14 describes the incidence of lung infection between groups by funnel plot. Circles of literature are found on both sides of the midline, and are nearly symmetrical, indicating no publication bias in included literature.

\section{Discussion}

At present, intracranial pressure monitoring is taken as one of the routine monitoring methods in modern guidelines for the diagnosis and treatment of brain injuries. Jahns et al. [2019] (16) found that patients with severe head injury receiving intracranial pressure monitoring and intensive care can reduce the fatality rate by more than $12 \%$. In addition, the implementation of intracranial pressure monitoring can early detect changes in intracranial disease, determines cerebral perfusion pressure and cerebral blood flow, guides clinical treatment, and determines the prognosis of patients early and treat them early (17). 


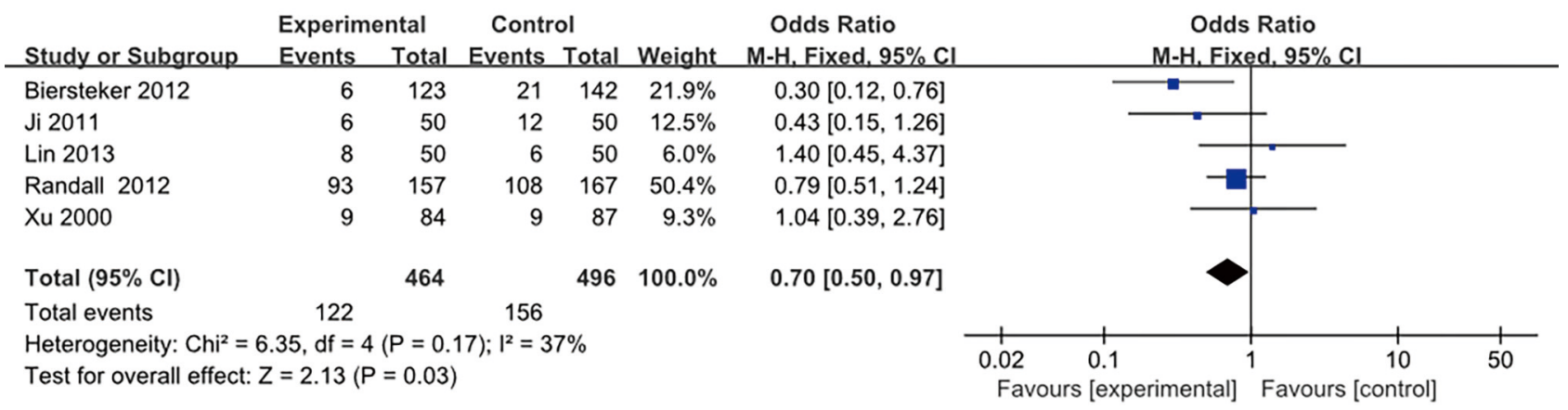

Figure 13 Incidence of infection between groups.

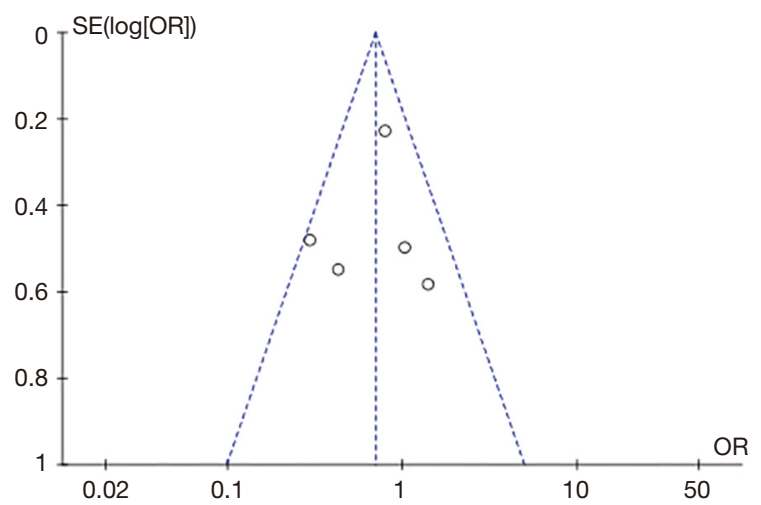

Figure 14 Funnel plot of infection incidence between groups.

Severe head injury and cerebrovascular disease are one of the important causes of death and disability, and elevated intracranial pressure is a common manifestation. Once it occurs, as the blood perfusion of the surviving brain tissue is reduced, the delivery of oxygen and metabolites is reduced, and the removal of metabolic waste and toxins is reduced, resulting in secondary brain damage $(15,18)$. At present, there are few studies on the correlation between intracranial pressure monitoring and the prognosis of patients with severe head injury, and there is no relevant theoretical basis. Therefore, the intracranial pressure monitoring was taken as an important monitoring method in this work, to study the impact of intracranial pressure monitoring on the prognosis of severe head injury through meta-analysis. Of the 13 articles included in this study, 12 used the randomized controlled grouping, 1 used the retrospective analysis, and while this brings some bias to the research, it has limited effect on the results of this study. A single sample may be unstable, and metaanalysis is implemented to quantitatively integrate each article, which avoids differences between articles caused by sampling from different populations. Moreover, it assigns different weights to the results based on sample size, which increases credibility of the conclusion (19). Due to the inclusion criteria imposed in this study, the number of included articles was limited, and in future analyses the sample size should be increased to prevent bias.

In this work, the compound logic retrieval method was adopted to include 13 articles reporting the use of non-intracranial pressure monitoring as a control to allow comparison with the effect of intracranial pressure monitoring on the prognosis of severe craniocerebral injury. While the results show there is no heterogeneous in-hospital mortality between groups $\left(\chi^{2}=0.76, \mathrm{I}^{2}=0 \%\right.$, $\mathrm{P}=0.98)$, the hospital mortality of experimental group was substantially lower relative to that of ctrl group $(\mathrm{Z}=3.69, \mathrm{P}=0.0002)$, suggesting that the implementation of intracranial pressure monitoring can effectively reduce the hospital mortality of patients. There is heterogeneity in the probability of a better functional prognosis between groups of patients $\left(\chi^{2}=8.01, \mathrm{I}^{2}=50 \%, \mathrm{P}=0.09\right)$. The probability of favorable functional prognosis in experimental group was dramatically higher relative to ctrl group $(\mathrm{Z}=2.48, \mathrm{P}=0.01)$, and the incidence of renal failure in experimental group was dramatically lower versus ctrl group $(\mathrm{Z}=2.71, \mathrm{P}=0.007)$. This is consistent with the findings of Stocchetti [2017] (20), indicating that intracranial pressure monitoring can effectively reduce in-hospital mortality and the incidence of infection in patients with severe craniocerebral injury and improve the functional prognosis of patients.

\section{Conclusions}

Boolean logic retrieval was adopted in this work. A control 
group extracted from the results of 13 articles that used non-intracranial pressure monitoring were included for meta-analysis to compare the effect of intracranial pressure monitoring on the prognosis of severe craniocerebral injury. The results show that intracranial pressure monitoring can effectively reduce in-hospital mortality and the incidence of infection in patients with severe craniocerebral injury and improve the functional prognosis of patients. However, there are still some shortcomings because of influence of various confounding factors. The selected works are casecontrol studies, with inherent survival bias. Moreover, many risk factors aren't considered, which greatly reduces the combined effect size. In the future, it will be considered to conduct follow-up for patients with severe head injury, to discover the effect of intracranial pressure monitoring on its prognosis.

\section{Acknowledgments}

Funding: None.

\section{Footnote}

Reporting Checklist: The authors have completed the PRISMA reporting checklist. Available at http://dx.doi. org/10.21037/apm-21-897

Conflicts of Interest: All authors have completed the ICMJE uniform disclosure form (available at http://dx.doi. org/10.21037/apm-21-897). The authors have no conflicts of interest to declare.

Ethical Statement: The authors are accountable for all aspects of the work in ensuring that questions related to the accuracy or integrity of any part of the work are appropriately investigated and resolved.

Open Access Statement: This is an Open Access article distributed in accordance with the Creative Commons Attribution-NonCommercial-NoDerivs 4.0 International License (CC BY-NC-ND 4.0), which permits the noncommercial replication and distribution of the article with the strict proviso that no changes or edits are made and the original work is properly cited (including links to both the formal publication through the relevant DOI and the license). See: https://creativecommons.org/licenses/by-nc$\mathrm{nd} / 4.0 \%$.

\section{References}

1. Lin D, Cai LF, Zhong LH, et al. Application study of early ventilation combined mild hypothermia hibernation on patients with severe traumatic brain injury. China Medical Herald 2013;10:51-53, 57.

2. Ji BY, Duan X, Miu N. Cerebral function monitoring by transcranial doppler combined with near-infrared spectroscopy during total aorta arch replacement procedure. Chinese Journal of Extracorporeal Circulation 2011;9:71-4.

3. Haddad S, Aldawood AS, Alferayan A, et al. Relationship between intracranial pressure monitoring and outcomes in severe traumatic brain injury patients. Anaesth Intensive Care 2011;39:1043-50.

4. Yu SL, Chen H, Ye ZH, et al. Application of continuous intracranial pressure monitoring in severe craniocerebral injury. Clinical Medicine of China 2011;27:612-4.

5. Xu R, Zhai CM. Significance of continuous dynamic intracranial pressure monitoring in severe craniocerebral injury. Journal of Postgraduates of Medicine 2000;23:36-7.

6. Mauritz W, Steltzer H, Bauer P, et al. Monitoring of intracranial pressure in patients with severe traumatic brain injury: an Austrian prospective multicenter study. Intensive Care Med 2008;34:1208-15.

7. Huang QB, Zhang Y, Su YX, et al. Prognostic correlation of intracranial pressure monitoring in patients with severe craniocerebral injury. Zhonghua Yi Xue Za Zhi 2013;93:1788-90.

8. Biersteker HA, Andriessen TM, Horn J, et al. Factors influencing intracranial pressure monitoring guideline compliance and outcome after severe traumatic brain injury. Crit Care Med 2012;40:1914-22.

9. Qiu BH, Qi ST, Zeng H, et al. Clinical study of continuous intracranial pressure monitoring to guide the treatment of severe craniocerebral injury. Chinese Journal of Neurosurgery 2013;29:933-6.

10. Griesdale DE, McEwen J, Kurth T, et al. External ventricular drains and mortality in patients with severe traumatic brain injury. Can J Neurol Sci 2010;37:43-8.

11. Kostić A, Stefanović I, Novak V, et al. Prognostic significance of intracranial pressure monitoring and intracranial hypertension in severe brain trauma patients. Med Pregl 2011;64:461-5.

12. Narayan V, Mohammed N, Savardekar AR, et al. Noninvasive Intracranial Pressure Monitoring for Severe Traumatic Brain Injury in Children: A Concise Update on 
Current Methods. World Neurosurg 2018;114:293-300.

13. Chesnut RM, Temkin N, Carney N, et al. A trial of intracranial-pressure monitoring in traumatic brain injury. N Engl J Med 2012;367:2471-81. Erratum in: N Engl J Med 2013;369:2465.

14. Nakhost A, Perry JC, Frank D. Assessing the outcome of compulsory treatment orders on management of psychiatric patients at $2 \mathrm{McGill}$ University-associated hospitals. Can J Psychiatry 2012;57:359-65.

15. Mauritz W, Steltzer H, Bauer P, et al. Monitoring of intracranial pressure in patients with severe traumatic brain injury: an Austrian prospective multicenter study. Intensive Care Med 2008;34:1208-15.

16. Jahns FP, Miroz JP, Messerer M, et al. Quantitative pupillometry for the monitoring of intracranial hypertension in patients with severe traumatic brain injury. Crit Care 2019;23:155.

17. Zhou J, Li J, Ye T, et al. Ultrasound measurements versus

Cite this article as: Wang $\mathrm{H}, \mathrm{He} \mathrm{Y}$, Liang $\mathrm{R}, \mathrm{Wu} \mathrm{X}$, Zhao L, Yang J, Tang X. A meta-analysis and systematic review of intracranial pressure monitoring on severe craniocerebral injury. Ann Palliat Med 2021;10(5):5380-5390. doi: 10.21037/ apm-21-897 invasive intracranial pressure measurement method in patients with brain injury: a retrospective study. BMC Med Imaging 2019;19:53.

18. Al Saiegh F, Philipp L, Mouchtouris N, et al. Comparison of Outcomes of Severe Traumatic Brain Injury in 36,929 Patients Treated with or without Intracranial Pressure Monitoring in a Mature Trauma System. World Neurosurg 2020;136:e535-41.

19. Smith M, Maas AIR. An algorithm for patients with intracranial pressure monitoring: filling the gap between evidence and practice. Intensive Care Med 2019;45:1819-21.

20. Stocchetti N, Zoerle T, Carbonara M. Intracranial pressure management in patients with traumatic brain injury: an update. Curr Opin Crit Care 2017;23:110-4.

(English Language Editor: B. Draper) 